\title{
ON RELATIVE COMMUTING PROBABILITY OF FINITE RINGS
}

\author{
PARAMA DUTTA AND RAJAT KANTI NATH
}

Received 11 March, 2017

\begin{abstract}
In this paper we study the probability that the commutator of a randomly chosen pair of elements, one from a subring of a finite ring and other from the ring itself is equal to a given element of the ring.
\end{abstract}

2010 Mathematics Subject Classification: 16U70; 16 U80.

Keywords: finite ring, commuting probability, $\mathbb{Z}$-isoclinism of rings

\section{INTRODUCTION}

Let $S$ be a subring of a finite ring $R$. The relative commuting probability of $S$ in $R$ denoted by $\operatorname{Pr}(S, R)$ is the probability that a randomly chosen pair of elements one from $S$ and the other from $R$ commute. That is

$$
\operatorname{Pr}(S, R)=\frac{|\{(x, y) \in S \times R: x y=y x\}|}{|S||R|} .
$$

This ratio $\operatorname{Pr}(S, R)$ can also be viewed as the probability that the commutator of a randomly chosen pair of elements, one from the subring $S$ and the other from $R$, equals the zero of $R$. We write $[x, y]$ to denote the commutator $x y-y x$ of $x, y \in R$. The study of $\operatorname{Pr}(S, R)$ was initiated in [2]. Note that $\operatorname{Pr}(R, R)$, also denoted by $\operatorname{Pr}(R)$, is the probability that a randomly chosen pair of elements of $R$ commute. The ratio $\operatorname{Pr}(R)$ is called the commuting probability of $R$ and it was introduced by MacHale [6] in the year 1976. It is worth mentioning that the commuting probability of algebraic structures was originated from the works of Erdös and Turán [4] in the year 1968.

In this paper we consider the probability that the commutator of a randomly chosen pair of elements, one from the subring $S$ and the other from $R$, equals a given element $r$ of $R$. We write $\operatorname{Pr}_{r}(S, R)$ to denote this probability. Therefore

$$
\operatorname{Pr}_{r}(S, R)=\frac{|\{(x, y) \in S \times R:[x, y]=r\}|}{|S||R|} .
$$

Clearly $\operatorname{Pr}_{r}(S, R)=0$ if and only if $r \notin K(S, R):=\{[x, y]: x \in S, y \in R\}$. Therefore we consider $r$ to be an element of $K(S, R)$ throughout the paper. Also $\operatorname{Pr}_{0}(S, R)=$ $\operatorname{Pr}(S, R)$ where 0 is the zero of $R$. It may be mentioned here that the case when 
$S=R$ is already considered in [3] by the authors. Interchanging $S$ and $R$ one may define $\operatorname{Pr}_{r}(R, S)$ for $r \in R$.

The aim of this paper is to obtain some computing formulas and bounds for $\operatorname{Pr}_{r}(S, R)$. We also discuss an invariance property of $\operatorname{Pr}_{r}(S, R)$ under $\mathbb{Z}$-isoclinism. The motivation of this paper lies in [7] where analogous generalization of commuting probability of finite group is studied.

We write $[S, R]$ and $[x, R]$ for $x \in S$ to denote the additive subgroups of $(R,+)$ generated by the sets $K(S, R)$ and $\{[x, y]: y \in R\}$ respectively. Note that $[x, R]=$ $\{[x, y]: y \in R\}$. Let $Z(S, R):=\{x \in S: x y=y x \forall y \in R\}$. Then $Z(R):=Z(R, R)$ is the center of $R$. Further, if $r \in R$ then the set $C_{S}(r):=\{x \in S: x r=r x\}$ is a subring of $S$ and $\cap_{r \in R} C_{S}(r)=Z(S, R)$. We write $\frac{R}{S}$ and $|R: S|$ to denote the additive quotient group and the index of $S$ in $R$ respectively.

\section{COMPUTING FORMULA FOR $\operatorname{Pr}_{r}(S, R)$}

In this section, we derive some computing formulas for $\operatorname{Pr}_{r}(S, R)$. We begin with the following useful lemmas.

Lemma 1 (Lemma 2.1 in [3]). Let $R$ be a finite ring. Then

$$
|[x, R]|=\left|R: C_{R}(x)\right| \text { for all } x \in R .
$$

Lemma 2. Let $S$ be a subring of a finite ring $R$ and $T_{x, r}(S, R)=\{y \in R:[x, y]=$ $r$ \} for $x \in S$ and $r \in R$. Then we have the followings

(1) $T_{x, r}(S, R) \neq \phi$ if and only if $r \in[x, R]$.

(2) If $T_{x, r}(S, R) \neq \phi$ then $T_{x, r}(S, R)=t+C_{R}(x)$ for some $t \in T_{x, r}(S, R)$.

Proof. Part (1) follows from the fact that $y \in T_{s, r}(S, R)$ if and only if $r \in[s, R]$. Let $t \in T_{x, r}(S, R)$ and $p \in t+C_{R}(x)$. Then $[x, p]=r$ and so $p \in T_{x, r}(S, R)$. Therefore, $t+C_{R}(x) \subseteq T_{x, r}(S, R)$. Again, if $y \in T_{x, r}(S, R)$ then $(y-t) \in C_{R}(x)$ and so $y \in t+C_{R}(x)$. Therefore, $T_{x, r}(S, R) \subseteq t+C_{R}(x)$. Hence part (2) follows.

Now we state and prove the following main result of this section.

Theorem 1. Let $S$ be a subring of a finite ring $R$. Then

$$
\operatorname{Pr}_{r}(S, R)=\frac{1}{|S||R|} \sum_{\substack{x \in S \\ r \in[x, R]}}\left|C_{R}(x)\right|=\frac{1}{|S|} \sum_{\substack{x \in S \\ r \in[x, R]}} \frac{1}{|[x, R]|} .
$$

Proof. Note that $\{(x, y) \in S \times R:[x, y]=r\}=\cup_{x \in S}\left(\{x\} \times T_{x, r}(S, R)\right)$. Therefore, by (1.1) and Lemma 2, we have

$$
|S||R| \operatorname{Pr}_{r}(S, R)=\sum_{x \in S}\left|T_{x, r}(S, R)\right|=\sum_{\substack{x \in S \\ r \in[x, R]}}\left|C_{R}(x)\right| .
$$


The second part follows from (2.1) and Lemma 1.

Proposition 1. Let $S$ be a subring of a finite ring $R$ and $r \in R$. Then $\operatorname{Pr}_{r}(S, R)=$ $\operatorname{Pr}_{-r}(R, S)$. However, if $2 r=0$ then $\operatorname{Pr}_{r}(S, R)=\operatorname{Pr}_{r}(R, S)$.

Proof. Let $X=\{(x, y) \in S \times R:[x, y]=r\}$ and $Y=\{(y, x) \in R \times S:[y, x]=$ $-r$ \}. It is easy to see that $(x, y) \mapsto(y, x)$ defines a bijective mapping from $X$ to $Y$. Therefore, $|X|=|Y|$ and the result follows from (1.1).

Second part follows from the fact that $r=-r$ if $2 r=0$.

Proposition 2. Let $S_{1}$ and $S_{2}$ be two subrings of the finite rings $R_{1}$ and $R_{2}$ respectively. If $\left(r_{1}, r_{2}\right) \in R_{1} \times R_{2}$ then

$$
\operatorname{Pr}_{\left(r_{1}, r_{2}\right)}\left(S_{1} \times S_{2}, R_{1} \times R_{2}\right)=\operatorname{Pr}_{r_{1}}\left(S_{1}, R_{1}\right) \operatorname{Pr}_{r_{2}}\left(S_{2}, R_{2}\right) .
$$

Proof. Let $X_{i}=\left\{\left(x_{i}, y_{i}\right) \in S_{i} \times R_{i}:\left[x_{i}, y_{i}\right]=r_{i}\right\}$ for $i=1,2$ and

$Y=\left\{\left(\left(x_{1}, x_{2}\right),\left(y_{1}, y_{2}\right)\right) \in\left(S_{1} \times S_{2}\right) \times\left(R_{1} \times R_{2}\right):\left[\left(x_{1}, x_{2}\right),\left(y_{1}, y_{2}\right)\right]=\left(r_{1}, r_{2}\right)\right\}$.

Then $\left(\left(x_{1}, y_{1}\right),\left(x_{2}, y_{2}\right)\right) \mapsto\left(\left(x_{1}, x_{2}\right),\left(y_{1}, y_{2}\right)\right)$ defines a bijective map from $X_{1} \times$ $X_{2}$ to $Y$. Therefore, $|Y|=\left|X_{1}\right|\left|X_{2}\right|$ and hence the result follows from (1.1).

Using Proposition 1 in Theorem 1, we get the following corollary.

Corollary 1. Let $S$ be a subring of a finite ring $R$. Then

$$
\operatorname{Pr}(R, S)=\operatorname{Pr}(S, R)=\frac{1}{|S||R|} \sum_{x \in S}\left|C_{R}(x)\right|=\frac{1}{|S|} \sum_{x \in S} \frac{1}{|[x, R]|} .
$$

We conclude this section with the following corollary.

Corollary 2. Let $S$ be a subring of a finite non-commutative ring $R$. If $|[S, R]|=$ $p$, a prime, then

$$
\operatorname{Pr}_{r}(S, R)= \begin{cases}\frac{1}{p}\left(1+\frac{p-1}{|S: Z(S, R)|}\right), & \text { if } r=0 \\ \frac{1}{p}\left(1-\frac{1}{|S: Z(S, R)|}\right), & \text { if } r \neq 0 .\end{cases}
$$

Proof. For $x \in S \backslash Z(S, R)$, we have $\{0\} \subsetneq[x, R] \subseteq[S, R]$. Since $|[S, R]|=p$, it follows that $[S, R]=[x, R]$ and hence $|[x, R]|=p$ for all $x \in S \backslash Z(S, R)$.

If $r=0$ then by Corollary 1, we have

$$
\begin{aligned}
\operatorname{Pr}_{r}(S, R) & =\frac{1}{|S|}\left(|Z(S, R)|+\sum_{x \in S \backslash Z(S, R)} \frac{1}{|[x, R]|}\right) \\
& =\frac{1}{|S|}\left(|Z(S, R)|+\frac{1}{p}(|S|-|Z(S, R)|)\right) \\
& =\frac{1}{p}\left(1+\frac{p-1}{|S: Z(S, R)|}\right) .
\end{aligned}
$$


If $r \neq 0$ then $r \notin[x, R]$ for all $x \in Z(S, R)$ and $r \in[x, R]$ for all $x \in S \backslash Z(S, R)$. Therefore, by Theorem 1, we have

$$
\begin{aligned}
\operatorname{Pr}_{r}(S, R) & =\frac{1}{|S|} \sum_{x \in S \backslash Z(S, R)} \frac{1}{|[x, R]|}=\frac{1}{|S|} \sum_{x \in S \backslash Z(S, R)} \frac{1}{p} \\
& =\frac{1}{p}\left(1-\frac{1}{|S: Z(S, R)|}\right) .
\end{aligned}
$$

Hence, the result follows.

\section{BOUNDS FOR $\operatorname{Pr}_{r}(S, R)$}

If $S$ is a subring of a finite ring $R$ then it was shown in [2, Theorem 2.16] that

$$
\operatorname{Pr}(S, R) \geq \frac{1}{|K(S, R)|}\left(1+\frac{|K(S, R)|-1}{|S: Z(S, R)|}\right) .
$$

Also, if $p$ is the smallest prime dividing $|R|$ then by [2, Theorem 2.5] and [2, Corollary 2.6] we have

$$
\operatorname{Pr}(S, R) \leq \frac{(p-1)|Z(S, R)|+|S|}{p|S|} \text { and } \operatorname{Pr}(R) \leq \frac{(p-1)|Z(R)|+|R|}{p|R|} .
$$

In this section, we obtain several bounds for $\operatorname{Pr}_{r}(S, R)$ and show that some of our bounds are better than the bounds given in (3.1) and (3.2). We begin with the following upper bound.

Proposition 3. Let $S$ be a subring of a finite ring $R$. If $p$ is the smallest prime dividing $|R|$ and $r \neq 0$ then

$$
\operatorname{Pr}_{r}(S, R) \leq \frac{|S|-|Z(S, R)|}{p|S|}<\frac{1}{p} .
$$

Proof. Since $r \neq 0$ we have $S \neq Z(S, R)$. If $x \in Z(S, R)$ then $r \notin[s, R]$. If $x \in S \backslash Z(S, R)$ then $C_{R}(x) \neq R$. Therefore, by Lemma 1 , we have $|[x, R]|=\mid R$ : $C_{R}(x) \mid>1$. Since $p$ is the smallest prime dividing $|R|$ we have $|[x, R]| \geq p$. Hence the result follows from Theorem 1.

Proposition 4. Let $S$ be a subring of a finite ring $R$. Then $\operatorname{Pr}_{r}(S, R) \leq \operatorname{Pr}(S, R)$ with equality if and only if $r=0$.

Proof. By Theorem 1 and Corollary 1, we have

$$
\operatorname{Pr}_{r}(S, R)=\frac{1}{|S||R|} \sum_{\substack{x \in S \\ r \in[x, R]}}\left|C_{R}(x)\right| \leq \frac{1}{|S||R|} \sum_{x \in S}\left|C_{R}(x)\right|=\operatorname{Pr}(S, R) .
$$

The equality holds if and only if $r=0$. 
Proposition 5. If $S_{1} \subseteq S_{2}$ are two subrings of a finite ring $R$ then

$$
\operatorname{Pr}_{r}\left(S_{1}, R\right) \leq\left|S_{2}: S_{1}\right| \operatorname{Pr}_{r}\left(S_{2}, R\right)
$$

Proof. By Theorem 1, we have

$$
\begin{aligned}
\left|S_{1}\right||R| \operatorname{Pr}_{r}\left(S_{1}, R\right)= & \sum_{\substack{x \in S_{1} \\
r \in[x, R]}}\left|C_{R}(x)\right| \\
& \leq \sum_{\substack{x \in S_{2} \\
r \in[x, R]}}\left|C_{R}(x)\right|=\left|S_{2}\right||R| \operatorname{Pr}_{r}\left(S_{2}, R\right) .
\end{aligned}
$$

Hence the result follows.

Note that equality holds in Proposition 5 if and only if $r \notin[x, R]$ for all $x \in S_{2} \backslash S_{1}$. If $r=0$ then the condition of equality reduces to $S_{1}=S_{2}$. Putting $S_{1}=S$ and $S_{2}=R$ in Proposition 5 we have the following corollary.

Corollary 3. If $S$ is a subring of a finite ring $R$ then

$$
\operatorname{Pr}_{r}(S, R) \leq|R: S| \operatorname{Pr}_{r}(R) .
$$

For any subring $S$ of $R$, let $m_{S}=\min \{|[x, R]|: x \in S \backslash Z(S, R)\}$ and $M_{S}=$ $\max \{|[x, R]|: x \in S \backslash Z(S, R)\}$. In the following theorem we give bounds for $\operatorname{Pr}(S, R)$ in terms of $m_{S}$ and $M_{S}$.

Theorem 2. Let $S$ be a subring of a finite ring $R$. Then

$$
\frac{1}{M_{S}}\left(1+\frac{M_{S}-1}{|S: Z(S, R)|}\right) \leq \operatorname{Pr}(S, R) \leq \frac{1}{m_{S}}\left(1+\frac{m_{S}-1}{|S: Z(S, R)|}\right) .
$$

The equality holds if and only if $m_{S}=M_{S}=|[x, R]|$ for all $x \in S \backslash Z(S, R)$.

Proof. Since $m_{S} \leq|[x, R]|$ and $M_{S} \geq|[x, R]|$ for all $x \in S \backslash Z(S, R)$, we have

$$
\frac{|S|-|Z(S, R)|}{M_{S}} \leq \sum_{x \in S \backslash Z(S, R)} \frac{1}{|[x, R]|} \leq \frac{|S|-|Z(S, R)|}{m_{S}} .
$$

Again, by Corollary 1, we have

$$
\operatorname{Pr}(S, R)=\frac{1}{|S|}\left(|Z(S, R)|+\sum_{x \in S \backslash Z(S, R)} \frac{1}{|[x, R]|}\right) .
$$

Hence, the result follows from (3.3) and (3.4).

Note that for any two integers $m \geq n$, we have

$$
\frac{1}{n}\left(1+\frac{n-1}{|S: Z(S, R)|}\right) \geq \frac{1}{m}\left(1+\frac{m-1}{|S: Z(S, R)|}\right) .
$$


Clearly equality holds in (3.5) if $Z(S, R)=S$. Further, if $Z(S, R) \neq S$ then equality holds if and only if $m=n$. Since $|K(S, R)| \geq M_{S}$, by (3.5), it follows that

$$
\frac{1}{M_{S}}\left(1+\frac{M_{S}-1}{|S: Z(S, R)|}\right) \geq \frac{1}{|K(S, R)|}\left(1+\frac{|K(S, R)|-1}{|S: Z(S, R)|}\right) .
$$

Therefore, the lower bound obtained in Theorem 2 is better than the lower bound given in (3.1) for $\operatorname{Pr}(S, R)$. Again, if $p$ is the smallest prime divisor of $|R|$ then $p \leq m_{S}$ and hence, by (3.5), we have

$$
\frac{1}{m_{S}}\left(1+\frac{m_{S}-1}{|S: Z(S, R)|}\right) \leq \frac{(p-1)|Z(S, R)|+|S|}{p|S|} .
$$

This shows that the upper bound obtained in Theorem 2 is better than the upper bound given in (3.2) for $\operatorname{Pr}(S, R)$.

Putting $S=R$ in Theorem 2 we have the following corollary.

Corollary 4. Let $R$ be a finite ring. Then

$$
\frac{1}{M_{R}}\left(1+\frac{M_{R}-1}{|R: Z(R)|}\right) \leq \operatorname{Pr}(R) \leq \frac{1}{m_{R}}\left(1+\frac{m_{R}-1}{|R: Z(R)|}\right) .
$$

The equality holds if and only if $m_{R}=M_{R}=|[x, R]|$ for all $x \in R \backslash Z(R)$.

We conclude this section noting that the lower bound obtained in Corollary 4 is better than the lower bound obtained in [2, Corollary 2.18]. Also, if $p$ is the smallest prime divisor of $|R|$ then the upper bound obtained in Corollary 4 is better than the upper bound given in (3.2) for $\operatorname{Pr}(R)$.

\section{4. $\mathbb{Z}$-ISOCLINISM AND $\operatorname{Pr}_{r}(S, R)$}

The idea of isoclinism of groups was introduced by Hall [5] in 1940. Years after in 2013, Buckley et al. [1] introduced $\mathbb{Z}$-isoclinism of rings. Recently, Dutta et al. [2] have introduced $\mathbb{Z}$-isoclinism between two pairs of rings, generalizing the notion of $\mathbb{Z}$-isoclinism of rings. Let $S_{1}$ and $S_{2}$ be two subrings of the rings $R_{1}$ and $R_{2}$ respectively. Recall that a pair of mappings $(\alpha, \beta)$ is called a $\mathbb{Z}$-isoclinism between $\left(S_{1}, R_{1}\right)$ and $\left(S_{2}, R_{2}\right)$ if $\alpha: \frac{R_{1}}{Z\left(S_{1}, R_{1}\right)} \rightarrow \frac{R_{2}}{Z\left(S_{2}, R_{2}\right)}$ and $\beta:\left[S_{1}, R_{1}\right] \rightarrow\left[S_{2}, R_{2}\right]$ are additive group isomorphisms such that $\alpha\left(\frac{S_{1}}{Z\left(S_{1}, R_{1}\right)}\right)=\frac{S_{2}}{Z\left(S_{2}, R_{2}\right)}$ and $\beta\left(\left[x_{1}, y_{1}\right]\right)=$ $\left[x_{2}, y_{2}\right]$ whenever $x_{i} \in S_{i}, y_{i} \in R_{i}$ for $i=1,2 ; \alpha\left(x_{1}+Z\left(S_{1}, R_{1}\right)\right)=x_{2}+Z\left(S_{2}, R_{2}\right)$ and $\alpha\left(y_{1}+Z\left(S_{1}, R_{1}\right)\right)=y_{2}+Z\left(S_{2}, R_{2}\right)$. Two pairs of rings are said to be $\mathbb{Z}$ isoclinic if there exists a $\mathbb{Z}$-isoclinism between them.

In [2, Theorem 3.3], Dutta et al. proved that $\operatorname{Pr}\left(S_{1}, R_{1}\right)=\operatorname{Pr}\left(S_{2}, R_{2}\right)$ if the rings $R_{1}$ and $R_{2}$ are finite and the pairs $\left(S_{1}, R_{1}\right)$ and $\left(S_{2}, R_{2}\right)$ are $\mathbb{Z}$-isoclinic. We conclude this paper with the following generalization of [2, Theorem 3.3]. 
Theorem 3. Let $S_{1}$ and $S_{2}$ be two subrings of the finite rings $R_{1}$ and $R_{2}$ respectively. If $(\alpha, \beta)$ is a $\mathbb{Z}$-isoclinism between $\left(S_{1}, R_{1}\right)$ and $\left(S_{2}, R_{2}\right)$ then

$$
\operatorname{Pr}_{r}\left(S_{1}, R_{1}\right)=\operatorname{Pr}_{\beta(r)}\left(S_{2}, R_{2}\right) .
$$

Proof. By Theorem 1, we have

$$
\operatorname{Pr}_{r}\left(S_{1}, R_{1}\right)=\frac{\left|Z\left(S_{1}, R_{1}\right)\right|}{\left|S_{1}\right|\left|R_{1}\right|} \sum_{\substack{x_{1}+Z\left(S_{1}, R_{1}\right) \in \frac{S_{1}}{Z\left(S_{1}, R_{1}\right)} \\ r \in\left[x_{1}, R_{1}\right]}}\left|C_{R_{1}}\left(x_{1}\right)\right|
$$

noting that $r \in\left[x_{1}, R_{1}\right]$ if and only if $r \in\left[x_{1}+z, R_{1}\right]$ and $C_{R_{1}}\left(x_{1}\right)=C_{R_{1}}\left(x_{1}+z\right)$ for all $z \in Z\left(S_{1}, R_{1}\right)$. Now, by Lemma 1 , we have

$$
\operatorname{Pr}_{r}\left(S_{1}, R_{1}\right)=\frac{\left|Z\left(S_{1}, R_{1}\right)\right|}{\left|S_{1}\right|} \sum_{\substack{x_{1}+Z\left(S_{1}, R_{1}\right) \in \frac{S_{1}}{Z\left(S_{1}, R_{1}\right)} \\ r \in\left[x_{1}, R_{1}\right]}} \frac{1}{\left|\left[x_{1}, R_{1}\right]\right|} .
$$

Similarly, it can be seen that

$$
\operatorname{Pr}_{\beta(r)}\left(S_{2}, R_{2}\right)=\frac{\left|Z\left(S_{2}, R_{2}\right)\right|}{\left|S_{2}\right|} \sum_{\substack{x_{2}+Z\left(S_{2}, R_{2}\right) \in \frac{S_{2}}{Z\left(S_{2}, R_{2}\right)} \\ \beta(r) \in\left[x_{2}, R_{2}\right]}} \frac{1}{\left|\left[x_{2}, R_{2}\right]\right|} .
$$

Since $(\alpha, \beta)$ is a $\mathbb{Z}$-isoclinism between $\left(S_{1}, R_{1}\right)$ and $\left(S_{2}, R_{2}\right)$ we have $\frac{\left|S_{1}\right|}{\left|Z\left(S_{1}, R_{1}\right)\right|}=$ $\frac{\left|S_{2}\right|}{\left|Z\left(S_{2}, R_{2}\right)\right|},\left|\left[x_{1}, R_{1}\right]\right|=\left|\left[x_{2}, R_{2}\right]\right|$ and $r \in\left[x_{1}, R_{1}\right]$ if and only if $\beta(r) \in\left[x_{2}, R_{2}\right]$. Hence, the result follows from (4.1) and (4.2).

\section{ACKNOWLEDGEMENT}

The authors would like to thank the referee for his/her valuable comments and suggestions.

\section{REFERENCES}

[1] S. M. Buckley, D. MacHale, and A. Ní Shé, "Finite rings with many commuting pairs of elements." Preprint.

[2] J. Dutta, D. K. Basnet, and R. K. Nath, "On commuting probability of finite rings.” Indag. Math., vol. 28, no. 2, pp. 372-382, 2017, doi: 10.1016/j.indag.2016.10.002.

[3] P. Dutta and R. K. Nath, "On $r$-commuting probability of finite rings." Preprint

[4] P. Erdös and P. Turán, "On some problems of a statistical group-theory. IV." Acta. Math. Acad. Sci. Hungar., vol. 19, pp. 413-435, 1968, doi: 10.1007/BF01894517.

[5] P. Hall, "The classification of prime power groups." J. Reine Angew. Math., vol. 182, pp. 130-141, 1940, doi: 10.1515/crll.1940.182.130.

[6] D. MacHale, "Commutativity in Finite Rings." Amer. Math. Monthly, vol. 83, pp. 30-32, 1976, doi: $10.1080 / 00029890.1976 .11994032$. 
[7] R. K. Nath and M. K. Yadav, "Some results on relative commutativity degree." Rend. Circ. Mat. Palermo, vol. 64, no. 2, pp. 229-239, 2015, doi: 10.1007/s12215-015-0194-x.

Authors' addresses

\section{Parama Dutta}

Tezpur University, Department of Mathematical Sciences, Napaam-784028, Sonitpur, Assam, India.

E-mail address: parama@gonitsora.com

Rajat Kanti Nath

Tezpur University, Department of Mathematical Sciences, Napaam-784028, Sonitpur, Assam, India.

E-mail address: rajatkant inath@yahoo.com 